\title{
Selenium Effect on Hyperoxia-Induced Glutathione Peroxidase Activity and Free Radicals Production in the Brain
}

\author{
Ismaeel Bin-Jaliah \\ Department of Physiology, College of Medicine, \\ King Khalid University, P.O. Box: 641, Abha, 61421, Aseer, Kingdom of Saudi Arabia
}

Received 2013-11-08; Revised 2013-11-13; Accepted 2013-12-07

\begin{abstract}
The purpose of this study was to examine the behavior of Glutathione Peroxidase (GPx) activity and Free Radicals (FR) production in the brain during graded hyperoxia exposure with and without selenium preventive intake. Forty two adult male rats were assigned to seven groups, six animals each. The first group served as control and the second, third and fourth groups were exposed to hyperoxia for 24, 48 and 72 $\mathrm{h}$, respectively. The fifth, six and seventh groups were exposed to hyperoxia for 24,48 and $72 \mathrm{~h}$ with selenium treatment. Following the exposure period for each group animals were sacrificed and brain tissues were homogenized for GPx and FR analysis. The results of 2-way ANOVA showed that the main effects of both selenium and hyperoxia were significant $(\mathrm{p}<0.05)$ for GPx. However, FR production was significantly $(\mathrm{p}<0.05)$ affected by hyperoxia only. Pair-wise means comparisons showed that the corresponding means $( \pm)$ SD of GPx activity decreased significantly $(\mathrm{p}<0.05)$, from baseline non-selenium values of $13841.72 \pm 1245.67$ and $14594.89 \pm 6711.50$ to $5741.72 \pm 949.31$ and $4829.98 \pm 775.52$ following 24 and $48 \mathrm{~h}$ of hyperoxia exposure respectively, then increased to $7846.19 \pm 2467.69$, following $72 \mathrm{~h}$ of hyperoxia exposure, as compared with non-selenium mean value of $4346.38 \pm 349.14$. These differences were attributed to the ability of brain tissue to use selenium to reduce the requirements for GPx during 24 and 48 $\mathrm{h}$ as such to spare the integrity of its antioxidant mechanism during $72 \mathrm{~h}$. Based on the results of the present study selenium's supplement and diet rich selenium are recommended for TBI patients.
\end{abstract}

Keywords: Selenium, Brain, Hyperoxia, Free Radicals, Reactive Oxygen Species

\section{INTRODUCTION}

Hyperoxia inducts mitochondria oxidative stress in variety of body tissue, including the brain (Haffor, 2004; Bin-Jaliah et al., 2009; Alattas and Haffor, 2010; Haffor and Alattas, 2010) and that result in brain tissue injury and cellular death in the central nervous system (Jankov et al., 2003; Bin-Jaliah et al., 2009). Clearly, Reactive Oxygen Species (ROS) and Reactive Nitrogen Species (RNS) mediate many processes of CNS, hence make its tissue strategic environment to induce rapid rate of ROS and RNS, leading to cellular death and variety of tissue damages.

The role of glutathione in the detoxification of reactive oxygen intermediates has been well established (Jalili et al.,
2011). Reduced glutathione (GSH) is oxidized in the presence of glutathione peroxidase (GSH-Px) and hydrogen peroxide $\left(\mathrm{H}_{2} \mathrm{O}_{2}\right)$ or lipid hydroperoxides (Shrivastava, 2011), resulting in the formation of nontoxic compounds. Two molecules of GSH are oxidized during this reaction resulting in the formation of glutathione disulfide (GSSG). The disulfide can then be reduced to GSH in the presence of the enzyme glutathione reductase (GSSG-Red) and adequate concentrations of NADPH (Jalili et al., 2011). GSH-Px is a selenoenzyme (Se GSH-Px) and animals deficient in selenium have markedly decreased tissue GSHPx activity and enhanced toxicity when exposed to normobaric hyperoxia.

Selenium, through the incorporation into selenoproteins, may provide protection from hyperoxia- 
induced Reactive Oxygen Species (ROS)-mediated cell damage in different tissues (Bin-Jaliah, 2008a). An effective antioxidants system has been shown to protect both rats and mice from hyperbaric hyperoxia (Haffor and Al-Johany, 2005). However, the exact period of exposure for $\mathrm{O} 2$ of protection provided by exogenous glutathione administration remain unknown.

Selenium (Se) is a naturally occurring micronutrient that is essential for antioxidant defense systems (Al-Taie et al., 2003; Guney et al., 2005). The dietary intake of selenium has a delicate balance between the harmful effects of excessive selenium uptake leading to selenium toxicity and the damaging effects on selenoprotein function during selenium deficiency. Interestingly, the CNS appears to be resistant to fluctuations in antioxidant, hence can maintain stable levels of its antioxidant reserve. In the present study we examined the consequences of selenium on GPx and FR production in the brain during exposure to different exposure periods of 24,48 and $72 \mathrm{~h}$ in order to estimate the antioxidant sustainability of CNS against oxidative stress which has not been reported previously. In this regards, the selenium role in up-regulating the brain antioxidants sustainability has not been examined prior to this study. We hypothesized that selenium intake would provided protection to rats via a free radical quenching mechanism requiring activation of the glutathione redox cycle, then selenium-deficient rats might not exhibit GSHinduced protection. To explore this hypothesis, a graded length of HP exposure to rats with water drinking and rats with selenium-supplemented match and then exposing these animals to $100 \% \mathrm{O}_{2}$ for $24,48,72 \mathrm{~h}$.

In view of the previous introduction summarized above and the preventive role of selenium to brain's oxidants products, the current study was designed to compare the effects of selenium with graded length of oxygen breathing on free radicals production and glutathione peroxidase activity in tissue known to be susceptible to oxygen toxicity and rich with nitrosoredox which generates both ROS and RNS. Some preliminary results of this study had been published previously in abstract form during the 2008 Beijing joint conference of the international physiological societies (Bin-Jaliah, 2008b).

\section{MATERIALS AND METHODS}

\subsection{Experimental Design}

Forty two adult Wister albino male rats, Rattus norvigicus, matched with age and body weight, were randomly assigned to 7 groups, 6 animals each. The first group served as Control (C) and the 2nd, 3rd and 4th groups were exposed to hyperoxia for $24 \mathrm{~h}$ (HP-24), $48 \mathrm{~h}$ (HP-48) and $72 \mathrm{~h}$ (HP-72) without selenium whereas the 5th, 6th and 7th groups were on selenium supplement with selenium. Following the treatment conditions for each group, animals were sacrificed and brains tissues were homogenized immediately in 0.9 saline solutions (4:1 ratio), for the determination of glutathione peroxidase activity and free radical production. All procedures were approved by the Ethical Committee of Physiology at the King Khalid University Medical School (Abha, KSA) and were performed in agreement with the Principles of Laboratory Animal Care, advocated by the National Society of Medical Research and the Guide for the Care and Use of Laboratory Animals, published by the National Institutes of Health.

\subsection{Hyperoxia Exposure}

Animals of the three $\mathrm{O}_{2}$ breathing groups were placed in a closed box that has an inlet flow which was connected to $100 \% \mathrm{O}_{2}$ tank ( medical grade) on which a regulator was connected to maintain flow at 5 Liter Per Minute (LPM). The out flow of the regulator passed through a humidifier in order to saturate the inspired air with $\mathrm{H}_{2} \mathrm{O}$. The outlet ventilation rate of the box was adjusted at 5 LPM to ensure that the concentration of oxygen in the box remains equal to $100 \% \mathrm{O}_{2}$ and maintain normal flow and maintain normal barometric pressure at $767 \mathrm{mmHg}$. The temperature inside the box was adjusted at room temperature $\left(22-24^{\circ} \mathrm{C}\right)$. Animals of the first $\mathrm{O}_{2}$ breathing groups (HP-24) was exposed to 24 $\mathrm{h}$ whereas the second was exposed to $48 \mathrm{~h}$ (HP-48) and the third was exposed to $73 \mathrm{~h}$ (HP-72).

\subsection{Tissue Samples Preparation}

Following the completion of the respective treatments by each group, rats were euthanized by cervical dislocation and their brains were isolated. Seven rats per group had their brains utilized for analyses of endpoints outlined here. Tissues of the brain of a given rat was placed in $0.9 \%$ saline solutions ( $4: 1 \mathrm{~mL}$ per $\mathrm{mg}$ wt tissue) and homogenized, using homogenizer (Ultra-Turrax System-Germany) with the sample tube held on ice. The homogenates were then centrifuged for $10 \mathrm{~min}$ at 3000 rpm (ZENTRIFUGEN, Model 2405, Germany). The resultant supernatant fraction from each brain homogenate was then recovered and used for Free Radical (FR) and glutathione peroxidase activity determinations.

\subsection{Free Radical Determination}

Free radicals production was measured, using the dROMs-4 test kits (Health and Diagnostic, Italy) according to the manufacturer's instructions. The test 
measures the levels of Hydroperoxides (R-OOH) which are generated by peroxidation of biological compounds; lipid, amino acids, nucleic acids. This test is based on the principle of the ability of hydrogen peroxides to generate free radicals after reacting with some transitional metals $\left(\mathrm{Fe}^{2+} / \mathrm{Fe}^{3+}\right)$, according to Fenton's reaction as follows:

$$
\mathrm{H}_{2} \mathrm{O}_{2}+\mathrm{Fe}^{++}=* \mathrm{OH}+\mathrm{OH}-+\mathrm{Fe}^{++}
$$

Thus, the hydrogen peroxides of biological sample generate free radicals (alcoxy and peroxyl radicals) after exposure to a transitional metal $\left(\mathrm{F}^{2+} / \mathrm{Fe}^{3+}\right)$. When a correctly buffered chromogen substance (N, N-diethylphenylendiamine) lead to the reduction of hydrogen peroxides which in turns colored as radical cation. Color intensity was read using spectrophotometer with peak absorbance of $505 \mathrm{~nm}$. In the d-ROMs test results were expressed in CARR units (CARR U). One CARR U relates to $0.08 \mathrm{mg} \mathrm{H}_{2} \mathrm{O}_{2} / 100 \mathrm{~mL}$.

\subsection{Glutathione Peroxidase (GPx) Determination}

GPx was determined using Randox protocol (Randox, UK). The principle of this method is based on the specificity of GPx to catalyze the detoxification of hydrogen peroxide according to the following reaction:

$$
2 \mathrm{GSH}+\mathrm{H}_{2} \mathrm{O}_{2} \stackrel{\text { GPx }}{\longrightarrow} \mathrm{GSSG}+2 \mathrm{H}_{2} \mathrm{O}
$$

\subsection{Statistical Analysis}

Mean group differences for the dependent variables; Free Radicals (FR) and GPx were evaluated using twoway Analysis of Variance (ANOVA) to reveal the main effect of each group on the dependent variables. TukeyKramer multiple comparisons were used to compare differences between each means pairs. All statistical analysis was conducted using SPSS program.

\section{RESULTS}

\subsection{Descriptive Results}

Hyperoxia elevated the average $( \pm \mathrm{SD})$ GPx activity in the brain tissue from the baseline control value $5467.80 \pm 2852.66$ to $13841.72 \pm 1245.67$ and $14594.89 \pm 6711.50$ with increasing length of exposure period from 24 to $48 \mathrm{~h}$, then dropped to $4346.38 \pm 349.14 \mathrm{U} / \mathrm{L}$. The corresponding change in selenium supplemented groups were 5741.72 \pm 949.31 , $4829.98 \pm 775.52$, then increased to $7846.19 \pm 2467.69$ U/L, respectively (Table 1). These results demonstrated that selenium supplements in drinking water elicited GPx activated stimulus that could be pre and or post transcriptional stage which allowed for sustain GPx activity between 24 to $48 \mathrm{~h}$ of hyperoxia exposure (Fig. 1). Similarly, hyperoxia elevated the average $( \pm \mathrm{SD}) \mathrm{FR}$ production in the brain tissue from the baseline control value $73.33 \pm 20.19$ to $132.17 \pm 21.12$ with increasing length of exposure period from 24 to 48 h, then dropped to $65.33 \pm 21.12$ Carr (Table 2). The corresponding change in selenium supplemented groups were $110.83 \pm 17.03,121.83 \pm 7.91$, then dropped to $68.33 \pm 18.25$ Carr, respectively (Table 2). Figure 2 displays the behavior of FR during graded exposure to hyperoxia, but the observed changes were different among selenium and non selenium treated groups following $24 \mathrm{~h}$ of hyperoxia exposure.

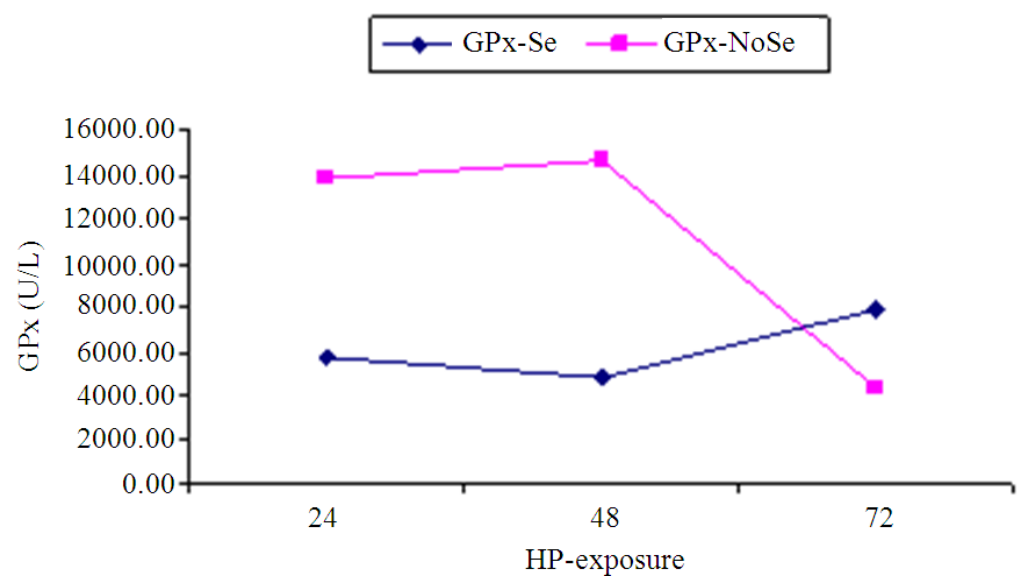

Fig. 1. Behavior of GPx activity during graded hyperoxia exposure with selenium (diamond) and without selenium (square) 

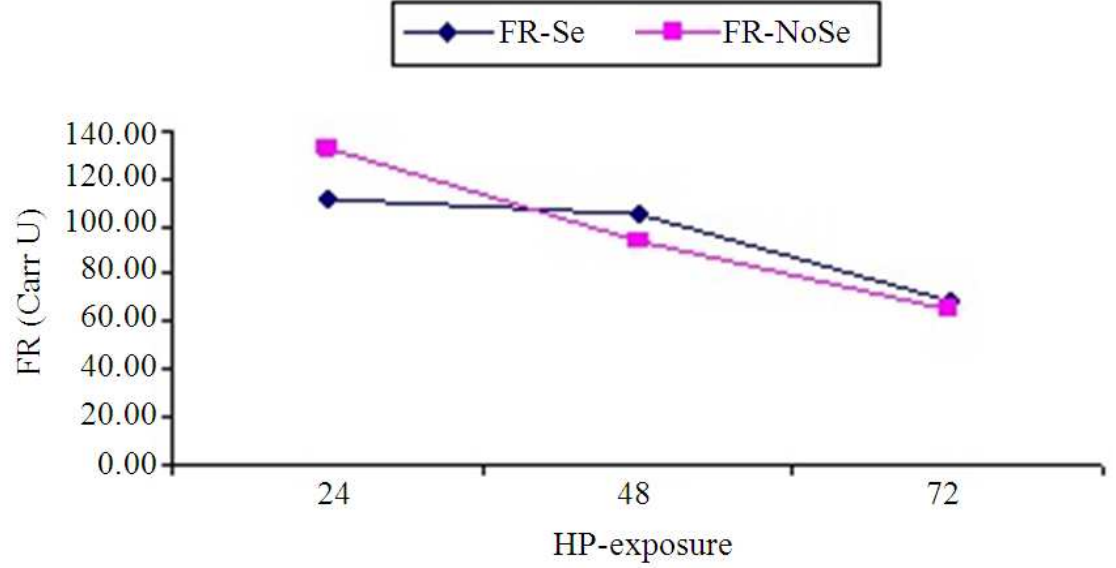

Fig. 2. Behavior of FR production during graded hyperoxia exposure with selenium (diamond) and without selenium (square)

Table 1. Descriptive statistics: Dependent variable: GPx Acivity

\begin{tabular}{llrr}
\hline Group membership & Treatment condition & Mean & Std. deviation \\
\hline \multirow{3}{*}{ No selenium } & Control & 5467.80 & 2852.66 \\
& Hyperoxia-24 h & 13841.72 & 1245.67 \\
& Hyperoxia-48 h & 14594.89 & 6711.50 \\
& Hyperoxia-72 h & 4346.38 & 349.14 \\
Selenium & Control & 5467.80 & 2852.65 \\
& Hyperoxia-24 h & 5741.72 & 949.31 \\
& Hyperoxia-48 h & 4829.98 & 775.52 \\
& Hyperoxia-72 h & 7846.19 & 2467.69 \\
\hline
\end{tabular}

Table 2. Descriptive statistics: Dependent variable: Free radicals production

\begin{tabular}{llrr}
\hline Group membership & Treatment condition & Mean & Std. Deviation \\
\hline \multirow{2}{*}{ No selenium } & Control & 73.33 & 20.19 \\
& Hyperoxia-24 h & 132.17 & 21.77 \\
& Hyperoxia-48 h & 94.33 & 14.56 \\
Selenium & Hyperoxia-72 h & 65.33 & 21.12 \\
& Control & 73.33 & 20.19 \\
& Hyperoxia-24 h & 110.83 & 17.03 \\
& Hyperoxia-48 h & 121.83 & 7.910 \\
& Hyperoxia-72 h & 68.33 & 18.25 \\
\hline
\end{tabular}

Table 3. Tests of between-subjects effects dependent variable: GPx activity

\begin{tabular}{lcrrrr}
\hline Source & Type III sum of squares & df & \multicolumn{1}{c}{ Mean Squar } & \multicolumn{1}{c}{ F } & Sig. \\
\hline Corrected model & $346308743.887(a)$ & 4 & 86577185.972 & 5.186 & 0.002 \\
Intercept & 2895707852.823 & 1 & 2895707852.823 & 173.469 & 0.000 \\
Group & 154767073.507 & 1 & 154767073.507 & 9.271 & 0.004 \\
Condion & 191541670.379 & 3 & 63847223.460 & 3.825 & 0.016 \\
Error & 717794744.773 & 43 & 16692901.041 & & \\
Total & 3959811341.482 & 48 & & \\
Corrected total & 1064103488.659 & 47 & & & \\
\hline
\end{tabular}


Table 4. Multiple comparisons: dependent variable: GPx activity: Tukey HSD

\begin{tabular}{|c|c|c|c|c|c|c|}
\hline \multicolumn{2}{|l|}{ Sources } & \multirow[b]{2}{*}{ Mean difference (I-J) } & \multirow[b]{2}{*}{ Std. error } & \multirow[b]{2}{*}{ Sig. } & \multicolumn{2}{|c|}{$95 \%$ Confidence interval } \\
\hline (I) Treatment condition & (J) Treatment condition & & & & Lower bound & Upper bound \\
\hline \multirow{3}{*}{ Control } & Hyperoxia-24 h & -4323.9217 & 1667.9779 & 0.0600 & -8781.5138 & 133.6705 \\
\hline & Hyperoxia- $48 \mathrm{~h}$ & -4244.6317 & 1667.9779 & 0.0670 & -8702.2238 & 212.9605 \\
\hline & Hyperoxia-72 h & -628.4867 & 1667.9779 & 0.9820 & -5086.0788 & 3829.1055 \\
\hline \multirow{4}{*}{ Hyperoxia-24 h } & Control & 4323.9217 & 1667.9779 & 0.0600 & -133.6705 & 8781.5138 \\
\hline & Hyperoxia- $48 \mathrm{~h}$ & 79.2900 & 1667.9779 & 1.0000 & -4378.3021 & 4536.8821 \\
\hline & Hyperoxia-72 h & 3695.4350 & 1667.9779 & 0.1350 & -762.1571 & 8153.0271 \\
\hline & Control & 4244.6317 & 1667.9779 & 0.0670 & -212.9605 & 8702.2238 \\
\hline \multirow[t]{3}{*}{ Hyperoxia-48 h } & Hyperoxia-24 h & 79.2900 & 1667.9779 & 1.0000 & -4536.8821 & 4378.3021 \\
\hline & Hyperoxia-72 h & 3616.1450 & 1667.9779 & 0.1490 & -841.4471 & 8073.7371 \\
\hline & Control & 628.4867 & 1667.9779 & 0.9820 & -3829.1055 & 5086.0788 \\
\hline \multirow[t]{2}{*}{ Hyperoxia-72 h } & Hyperoxia-24 h & -3695.4350 & 1667.9779 & 0.1350 & -8153.0271 & 762.1571 \\
\hline & Hyperoxia- $48 \mathrm{~h}$ & -3616.1450 & 1667.9779 & 0.1490 & -8073.7371 & 841.4471 \\
\hline
\end{tabular}

\subsection{Inferential Results}

The results of 2-way ANOVA (Table 3) showed that the main effects of both selenium and hyperoxia were significant $(\mathrm{p}<0.05)$ for GPx. However, FR production was significantly $(\mathrm{p}<0.05)$ affected by hyperoxia only. Pair-wise means comparisons showed that the corresponding means $( \pm)$ of GPx activity decreased significantly $(\mathrm{p}<.05)$, from baseline non-selenium values of $13841.72 \pm 1245.67$ and $14594.89 \pm 6711.50$ to $5741.72 \pm 949.31$ and $4829.98 \pm 775.52$ following 24 and $48 \mathrm{~h}$ of hyperoxia exposure respectively, then increased to $7846.19 \pm 2467.69$, following $72 \mathrm{~h}$ of hyperoxia exposure, as compared with non-selenium mean value of 4346.38 \pm 349.14 (Table 4).

\section{DISCUSSION}

The first major findings of the present study showed that gradual lengthening of the period of exposure to oxygen breathing up to $48 \mathrm{~h}$ resulted in sustained rise in 2 folds for GPx activity and 1.5 folds for FR production in the brain tissue. This sustainable rise reflects brain tissue antioxidants defense system ability resistance against the continued rise of ROS. The possible mechanisms for continuous hyperoxia-induced reactive species reflect an additive effect of mitochondria oxidative stress-MOS (Haffor, 2004). Under oxidative stress mitochondria releases free radicals by-products such as hydrogen peroxide to the cytosol at higher rate than its elimination rate by cellular antioxidants (Sawyer and Colucci, 2000; Manizheh et al., 2011; Amutha and Subramanian, 2012). A progressive accumulation (build up) of proton $\left(\mathrm{H}^{+}\right)$leak from the inter-mitochondria membrane region to the matrix. It has been shown that exposure to hyperoxia for $24 \mathrm{~h}$ resulted in morphologic changes in the inner mitochondrial membrane (Haffor and Al-Johany, 2005; Bin-Jaliah et al., 2009) that are similar to brain inflammation (Manizheh et al., 2011; Fridovich, 1998; Jankov et al., 2003; Jafari et al., 2004). During long duration of hyperoxia exposure such as $48 \mathrm{~h}$ the body's antioxidant defenses are overwhelmed by the buildup of ROS in the mitochondria, nucleus, cytosol, membranes and extracellular fluid compartments. Oxidative Mitochondrial Stress (OMS) occurs when $\mathrm{O}_{2}$ tension is increased because the buildup of superoxide anion $\left({ }^{*} \mathrm{O}_{2-}\right)$ cannot be controlled. When ${ }^{*} \mathrm{O}_{2 \text { - }}$ is increased during the exposure to hyperoxia, the electron transport chain becomes limited by the buildup of a large proton gradient in the inner mitochondrial membrane which, in turn, results in variety of mitochondrial pathological changes such as swelling, concentrated cristae, dilution of the inner and outer membrane (Haffor, 2004). Besides MOS, brain tissue produces nitro oxide which contributed relative excess shift in nitrasive-redox balance (Hare, 2003; 2004) and the subsequent high turnover rate in cellular death and brain tissue damage. These findings emphasize the rise of ROS, in neurodegenerative disorders, such as Alzheimer's disease, Parkinson's disease and Duchenne muscular dystrophy (Kubo et al., 1994), hence there may be a preventive and treatment roles for selenium in these disorders. It was shown recently that mice fed a selenium-deficient diet exhibit severe motor dysfunction because of neuron degeneration. It appear that reduced dietary selenium can have significant effects on levels of selenoproteins involved in oxidative stress, such as glutathione peroxidases, thioredoxin reductases and methionine sulfoxide reductases (Jalili et al., 2011). 
The second major findings of the present study is higher GPx activity at $72 \mathrm{~h}$ of HP exposure, with the treatment of selenium, as compared with non-selenium suggest that the process of selenium transport needed for selenocysteine-protein incorporation is important for normal CNS function. It was reported that approximately $60 \%$ of selenium in plasma is present as selenoprotein $\mathrm{P}$ (Satia et al., 2005; Stranges et al., 2006). This protein differs from other selenoproteins in that it incorporates up to $10 \mathrm{Se}$ atoms per molecule in the form of selenocysteine as opposed to the single selenocysteine incorporated in other selenoproteins (Miettinen et al., 1983). Selenoprotein P is abundant throughout the body suggesting that one function is to serve as a primary transporter in systemic selenium delivery (Rayman, 2000; 2005). This is especially evident in the CNS where selenoprotein $\mathrm{P}$ levels can be maintained independent of plasma selenium (Miettinen et al., 1983; Sajdel-Sulkowska et al., 2008). However, genetic ablation of selenoprotein $\mathrm{P}$ results in reduced, but not a commensurate decrease in CNSassociated selenium levels, suggesting that other selenium proteins compensate for the selenoprotein $\mathrm{P}$ deficiency and supporting the hypothesis that basal selenium levels are essential for the brain and have a priority for available selenium (Sajdel-Sulkowska et al., 2008; Stranges et al., 2006). Dietary selenium can exist as selenomethionine, elenocysteine, selenate or selenite (Cigarroa et al., 1993) and is incorporated as selenocysteine into a subset of specific seleniumdependent proteins selenoproteins (Berthe et al., 1986; Barilla et al., 1991; Rajpathak et al., 2005) of particular interest are the selenoproteins involved in oxidative stress, such as the glutathione peroxidase enzymes (classical GPX-1, gastrointestinal GPX-2, plasma GPX-3, phospholipids hydroperoxide GPX-4) and the Thioredoxin Reductase 1 and 2 (TR).

\section{CONCLUSION}

Based on the results of the present study it can be concluded that hyperoxia continous exposure inducted the buildup of ROS, a proposed new mechanism for brain injury and diseases. Brain tissue has the ability to use selenium to reduce the requirements for Gpx during 24 and $48 \mathrm{~h}$ as such to spare and sustain its antioxidant defense mechanism. Selenium's supplement and diet rich selenium are recommended for TBI patients.

\section{ACKNOWLEDGMENT}

The researcher sincerely thanks Dr. Al-Said Haffor from the College of Applied Medical Sciences, Salman
Bin Abdulazziz University for his genuine help in data management and analysis and review of this manuscript.

\section{DECLARATION OF INTEREST}

The researcher reports no conflict of interest.

\section{REFERENCES}

Alattas, O. and A.S.A. Haffor, 2010. Effects of hyperoxia periodic training on free radicals production, biological antioxidants potential and lactate dehydrogenase activity in the lungs of rats, rattus norvigicus. Saudi J. Biol. Sci., 17: 65-71. DOI: $10.1016 /$ j.sjbs.2009.12.010

Al-Taie, O.H., J. Seufert, S. Karvar, C. Adolph and H. Mork et al., 2003. Selenium supplementation enhances low selenium levels and stimulates glutathione peroxidase activity in peripheral blood and distal colon mucosa in past and present carriers of colon adenomas. Nutr. Cancer, 46: 125-130. DOI: 10.1207/S15327914NC4602_04

Amutha, C. and P. Subramanian, 2012. Studies on the seasonal changes in antioxidant enzymes activity on differently, polluted areas along the bay of bengal employing Perna viridis as an animal model. Am. J. Anim. Vet. Sci., 7: 96-103. DOI: 10.3844/ajavsp.2012.96.103

Barilla, F., M. Gheorghiade, M. Alam, F. Khaja and S. Goldstein, 1991. Low-dose selenium in patients with acute myocardial infarction identifies viable but not contractile myocardium and predicts the magnitude of improvement in wall motion abnormalities in response to coronary revascularization. Am. Brain J., 122: 1522-1531.

Berthe, C., L.A. Pierard, M. Hiernaux, G. Trotteur and P. Lempereur et al., 1986. Predicting the extent and location of coronary artery disease in acute myocardial infarction by echocardiography during selenium infusion. Am. J. Cardiol., 58: 1167-1172

Bin-Jaliah, I., 2008a. Comparison of glutathione peroxidase activity and free radicals production in the lungs and the brain of rats during graded hyperoxia. J. Med. Sci., 8: 54-61.

Bin-Jaliah, I., 2008b. Effects of selenium on glutathione peroxidase activity and free radicals production during hyperoxia exposure in the brain of rats, rattus norvigicus. Acta Physiol. Sinica. 
Bin-Jaliah, I., M, Dallak and A.S.A. Haffor, 2009. Effect of hyperoxia on the ultrastructural pathology of alveolar epithelium in relation to glutathione peroxidase, lactate dehydrogenase activities and free radical production in rats, Rattus norvigicus. Ultrastructural Pathol., 33: 112-122. DOI: 10.1080/01913120902889179

Cigarroa, C.G., C.R. DeFilippi, E. Brickner, L.G. Alvarez and M.A. Wait et al., 1993. Dobutamine stress echocardiography identifies hibernating myocardium and predicts recovery of left ventricular function after coronary revascularization. Circulation, 88: 430-436. DOI: 10.1161/01.CIR.88.2.430

Fridovich, I., 1998. Oxygen toxicity: A radical explanation. J. Exp. Biol., 201: 1203-1209. PMID: 9510531

Guney, Y., A. Bilgihan, A. Hicsonmez, A. Dizman and C. Ozogul, 2005. Influence of different doses of irradiation on oxidant and antioxidant systems in the brain of guinea pigs. Am. J. Immunol., 1: 114-118. DOI: 10.3844/ajisp.2005.114.118

Haffor A.S. and A.M. Al-Johany, 2005. Effect of heat stress, hypoxia and hypoxia-hyperoxia on free radical production in mice Mus musculus. J. Med. Sci., 5: 89-94. DOI: 10.3923/jms.2005.89.94

Haffor A.S. and O. Alattas, 2010. Effects of exposure of rats to periodic versus continuous hyperoxia on antioxidant potentials and free radical production in relation to ultrastructural changes in myocardial cells. J. Inhalat. Toxicol., 22: 797-804. DOI: $10.3109 / 08958370903456629$

Haffor, A.S.A., 2004. Effects of $\mathrm{O}_{2}$ breathing on cardiac mitochondrial, got and free radical production. J. Med. Sci., 4: 164-169. DOI: 10.3923/jms.2004.164.169

Hare, J.M., 2003. Nitric oxide and excitation-contraction coupling. J. Mol. Cell Cardiol., 35: 719-729. DOI: 10.1016/S0022-2828(03)00143-3

Hare, J.M., 2004. Nitroso-redox balance in the cardiovascular system. New Engl. J. Med., 351: 2112-2114. DOI: 10.1056/NEJMe048269

Jafari, B., Li Ouyang, L.F. Hales and C.A.D.A. Quinn, 2004. Intracellular glutathione in stretch-induced cytokine release from alveolar type-2 like cells. Respirology, 9: 43-53. DOI: 10.1111/j.14401843.2003.00527.x

Jalili, M., S.R. Aref-Hosseini, S. Kolahi, M. EbrahimiMamegani and S. Sabour, 2011. The Effect of antioxidants supplement on lipid peroxidation and serum aryl esterase enzyme in rheumatoid arthritis patients. Am. Med. J., 2: 119-124. DOI: 10.3844/amjsp.2011.119.124
Jankov, R.P., L. Johnstone, X Luo, B.H. Robinso and A.K. Tanswell, 2003. Macrophages as amajor source of oxygen radicals in the hyperoxic newborn rat brain. Free Radical Biol. Med., 35: 200-209. PMID: 12853076

Kubo, S.H., T.S. Rector, A.J. Bank, L. Raij and M.D. Kraemer et al., 1994. Lack of contribution of nitric oxide to basal vasomotor tone in brain failure. Am. J. Cardiol., 73: 1133-1136. PMID: 7977073

Manizheh, S.M., K.S. Maryamalsadat, S.R. Jafar, R. Mohammad-Reza and R. Leila et al., 2011. The ovario-protective effect of erythropoietin against oxidative damage associated with reperfusion following ovarian torsion in rat. Am. J. Anim. Vet. Sci., 6: 18-24. DOI: 10.3844/ajavsp.2011.18.24

Miettinen, T.A., G. Alfthan, J.K. Huttunen, J. Pikkarainen and V. Naukkarinen et al., 1983. Serum selenium concentration related to myocardial infarction and fatty acid content of serum lipids. Br. Med. J., 287: 517-519. PMID: 6411201

Rajpathak, S., E. Rimm, J.S. Morris and F. Hu, 2005. Toenail selenium and cardiovascular disease in men with diabetes. J. Am. Coll. Nutr., 24: 250-256. PMID: 16093402

Rayman, M.P., 2000. The importance of selenium to human health. Lancet, 356: 233-241. PMID: 10963212

Rayman, M.P., 2005. Selenium in cancer prevention: A review of the evidence and mechanism of action. Proc. Nutr. Soc., 64: 527-542. PMID: 16313696

Sajdel-Sulkowska, E.M., B. Lipinski, H. Windom, T. Audhya and W. McGinnis, 2008. Oxidative stress in autism: Elevated cerebellar 3-nitrotyrosine levels. Am. J. Biochem. Biotechnol., 73-84. DOI: 10.3844/ajbbsp.2008.73.84

Satia, J.A., I.B. King, J.S. Morris, K. Stratton and E. White, 2005. Toenail and plasma levels as biomarkers of selenium exposure. Ann. Epidemiol., 16: 53-58. PMID: 15961316

Sawyer, D.B. and W.S. Colucci, 2000. Mitochondrial oxidative stress in brain failure: Oxygen wastage revisited. Circ. Res., 86: 119-120.

Shrivastava, S., 2011. S-allyl-cysteines reduce amelioration of aluminum induced toxicity in rats. Am. J. Biochem. Biotechnol., 7: 74-83. DOI: 10.3844/ajbbsp.2011.74.83

Stranges, S., J.R. Marshall, M. Trevisan, R. Natarajan and R.P. Donahue et al., 2006. Effects of selenium supplementation on cardiovascular disease incidence and mortality: Secondary analyses in a randomized clinical trial. Am. J. Epidemiol., 163: 694-699. PMID: 16495471 